\title{
Structure and Development of the Attractive and Digestive Glands in the Carnivorous Pitcher Plant Nepenthes alata
}

\author{
T. Page Owen ${ }^{1}$, Alison Carini ${ }^{1}$, Lesley Sutherland ${ }^{1,2}$, Cara Hass $^{1,3}$, and Kyra Gabow ${ }^{1}$ \\ 1. Department of Botany, Connecticut College, New London, CT \\ 2. Current address: University of Maryland School of Medicine,Baltimore,MD \\ 3 . Current address: Boston Children's Hospital, Boston, MA
}

Carnivorous plants have evolved diverse structures to attract, capture and digest insects to permit plant growth in nutrient deficient substrates. Members of the genus Nepenthes utilize a passive pitfall mechanism to trap prey. Insects are attracted to the pitcher by sugary secretions from the nectary glands in the peristome region lining the trap opening. This type of trapping method is accomplished through three integral features. First, after entering the pitcher, the arthropod's ability to escape is obstructed by the inner protruding lip of the peristome (Fig. 1). Second, the surface of the peristome is covered by overlapping epidermal cells, directed towards the inner lumen of the pitcher (Fig. 2). When wet, the patterning of the peristome epidermal cells creates a slippery surface that interferes with the adherence of insect feet [1]. Third, below the peristome is an internal, slippery wax layer that also reduces the insect's ability to climb out of the pitcher. SEM observations found substantial variation of the peristome (Fig. 3) and wax deposits among the seven species examined: $N$. truncata, N. maxima, $N$. ventricosa, $N$. rafflesiana, $N$. hirsuta, $N$. ephippiata, and N. albomarginata.

Captured insects are digested and dissolved ions absorbed by multicellular glands at the base of the pitchers. Gland diameters varied from $120-400 \mu \mathrm{m}$ across species. It is also interesting to note that gland diameter did not necessarily correlate with the overall size of the pitcher. Like diameter, the degree of glandular lip coverage varied greatly among species. The glands from some species, such as $N$. truncata, had no lip coverage where as many of the species had glands with moderate glandular lip coverage, common to the "typical" Nepenthes gland structure. Other species such as $N$. ventricosa and $N$. rafflesiana, contained glands with no lip structure.

A focus of this study was the development of the nectary gland in $N$. alata. All ages of nectary glands had numerous organelles present consistent with a high level of molecular activity. These included numerous Golgi, elliptical mitochondria, endoplasmic reticulum, as well as underdeveloped plastids with starch grains occasionally present. Large nuclei were visible, with a large nucleoli present in most nuclei of most gland cells. The cytoplasm was electron-dense and grainy. Vacuoles were also frequently present, with electron-dense polyphenolics of varying amounts. However, the vacuoles in the nectary gland cells were small compared to the vacuoles of the surrounding tissue. The cell membrane remained close to the cell wall even when small protrusions were observed in some size stages. The cell wall was relatively thick, and there was little space between cells.

As the pitchers matured into an open, functional trap, the nectary glands had irregularly shaped secondary cell wall structures protruding into the plasma membrane in some of the nectary cells, as well as numerous plasmodesmata. These structures were not as common in basal gland cells. Definite cell wall protrusions were visible and common among the outer epidermal layer of nectary gland cells, especially in the exposed epithelial cells bordering the cuticle of the gland. We have started experiments 
to immunolocalize $\mathrm{H}^{+}$-ATPase proteins in the nectary cells to better understand developmental changes corresponding to secretory function. We are particularly interested if the pump co-localizes to the wall protuberances in the discrete areas of the gland [2].
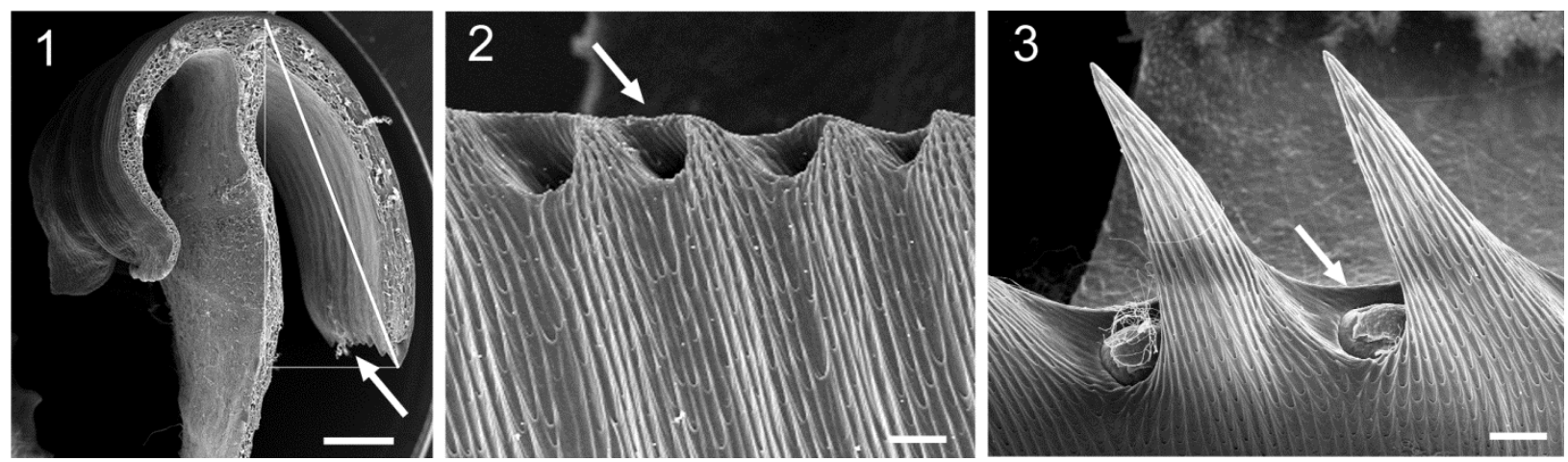

Figure 1. SEM image of longitudinal section of pitcher lip (peristome) of $N$. ventricosa. Nectary glands (arrow) face the inside of the pitcher; diagonal line illustrates measurement distance (bar $=1 \mathrm{~mm}$ ). Fig. 2. SEM image of N. maxima parallel to peristome surface with numerous nectary openings (arrow; bar $=100 \mu \mathrm{m}$ ) Fig. 3. $N$. ventricosa sample showing elongated "teeth" between nectary openings (arrow; bar $=100 \mu \mathrm{m})$.

\section{References:}

[1] HF Bohn and W Federle, Proc. Natl. Acad. Sci. USA 101 (2004), p. 14138.

[2] The authors acknowledge the contributions of Hope Barone, Jacquelyn Durand, Jeffrey Sumner and Daniel Smoot to this work. 